\title{
The Good, the Bad and the Indispensable - Insights into the Practical Potential of Emergency Response Information Systems and Drones for Firefighters
}

\author{
Julian Weidinger \\ Chair of Industrial Information \\ Systems, University of Bamberg \\ julian.weidinger@uni- \\ bamberg.de
}

\author{
Sebastian Schlauderer \\ Chair of Industrial Information \\ Systems, University of Bamberg \\ sebastian.schlauderer@uni- \\ bamberg.de
}

\author{
Sven Overhage \\ Chair of Industrial Information \\ Systems, University of Bamberg \\ sven.overhage@uni- \\ bamberg.de
}

\begin{abstract}
The introduction of innovative information technologies is frequently pursued to improve the on-site decision-making and hence the effectiveness of emergency response processes. Yet, the practical potential of innovative firefighter information technologies hardly has been investigated so far. In this paper, we present the results of a study, in which we interviewed 21 members of German fire departments about the potential of emergency response information systems and drones. The results suggest that firefighters find both technologies to deliver potential improvements. They also pointed to several possible drawbacks and critical requirements, however. The results of our study do not only provide a multifaceted overview of the potential benefits and risks that ought to be taken into account when introducing emergency response information systems or drones for firefighters. They also call for a systematic investigation of the practical potential of firefighter information technologies in general.
\end{abstract}

\section{Introduction}

As a result of the ongoing rapid technological progress, several innovative information technologies have been suggested to better support the emergency response operations of firefighters. Emerging technologies such as drones, robots, smart clothing, or indoor navigation approaches are supposed to support contextdependent on-site decisions with new sources of realtime information and hence bear a significant potential to change traditional emergency response processes. Both in scientific and practitioner literature, it is often assumed that the adoption and use of such technologies will increase the efficacy of emergency responses.

However, the adoption and use of new information technologies also introduce additional complexities for the firefighters who operate and maintain them on site. Any gain in functionality will thus have to be weighed against the additional overload or restrictions that arise for the users. Despite this challenge, the acceptance of innovative information technologies for firefighters has hardly been in the focus of research so far. As many of the proposed technologies moreover are not yet widespread in practice, little is known about their definite potential to support the on-site decision making and to increase the efficacy of emergency responses.

With the study presented in this paper, we intend to gain insights into the practical potential of innovative firefighter information technologies (FITs). To narrow its scope, we decided to examine the practical potential of unmanned aerial vehicles (UAVs, or drones) and emergency response information systems (ERIS), which are currently discussed in literature as two innovative FITs. In particular, we address the following research questions: "What is the practical potential of innovative firefighter technologies? Which factors increase or limit their potential in practice?"

To examine both research questions, we adopted a qualitative, interview-based research design. Such a design allows us to gain rich insights into the context and the reasons behind the results. Following this goal, we interviewed 21 members of German fire departments (FDs) about the potential of UAVs and ERIS. In an exploratory manner, we interpreted the results and derived conclusions regarding the factors that increase or limit the potential of the examined FITs in practice. Note that we did not specifically examine the potential of the two FITs to support extraordinary scenarios such as major disasters, but analyzed their ability to support the practices of firefighters in general. In so doing, we gained broader insights and were also able to study the potential of the FITs to support the daily routines of firefighters.

The findings of our research contribute to explaining the practical potential of emerging FITs. Emergency response processes have specific characteristics regarding time, effort, and complexity. If such characteristics are 
not identified and taken into account from the beginning, FITs run a risk of missing the needs of the users. In this context, the results of our study can provide an initial step to develop specific theories that explain the adoption of FITs. We proceed as follows: in section 2, we discuss the background and related work. In section 3 , we describe our research approach in detail. The results of our study are presented and discussed in section 4. We conclude by summarizing key findings and giving an outlook on future research in section 5 .

\section{Background and Related Work}

During the response to an emergency, firefighters make time-critical, context-dependent decisions on site. Their quality depends on the commanders' situation awareness [1]. The availability of information hence is one of the major determinants of a successful operation [2, 3]. Typically, however, commanders only have limited information about the status of casualties, the conditions inside a burning house, the status of responding units, etc. Consequently, decisions often have to be made with a high level of uncertainty and risk. To improve the situation awareness of firefighters, several new FITs have been proposed in academia and practice.

\subsection{Information Technologies for Firefighters}

To get an overview of novel FITs, we reviewed the literature following Webster's and Watson's [4] guidelines. We queried several databases including Google Scholar, AIS Library, IEEE Xplore, and the ACM Digital Library using keywords such as "firefighter”, "fire brigade”, “fire department”, or "firemen” together with “information system”, “information technology”, or specific keywords like "UAV”. We inspected the titles and abstracts of the resulting articles to eliminate irrelevant results. The remaining articles were analyzed using a narrative review method [5]. We also conducted backward and forward searches. Note that we did not include articles in practitioner outlets as they lack scientific rigor and rather provide anecdotal evidence.

The results of our review show that considerable work in the field is devoted to the analysis of and the response to large-scale disasters [6-11]. In recent years, especially social media and the inclusion of citizens into the response process were discussed [12-15]. Such approaches concentrate on the use of technologies in the specific event of major disasters, though. In contrast, we examine the use of FITs in general and hence also focus on their ability to facilitate the daily work of firefighters.

There also exist articles that treat FITs to support daily routines [16-20]. These articles typically concentrate on introducing specific FITs, however. In addition, they are typically technology-driven in nature. This means that they focus on proposing new technologies and discussing their theoretical potential based on their features. Usually, they do not examine how firefighters perceive such innovative technologies in practice. Literature on situation awareness shows that, in general, the introduction of additional information technologies might be beneficial. It also provides indications that the situation awareness can be hampered due to additional complexities and other disadvantages, though [21, 22].

Despite the unclear effects of novel FITs, we found only one article that analyzes their potential and specifically studies their perception in practice. In this article, six types of emerging FITs were identified [23]: digital plans/guides, ERIS, UAVs, unmanned ground vehicles, intelligent protective clothing, and indoor positioning. In a survey with over 900 responses, many of these technologies received a feedback that was contrary to the expectations in literature. Moreover, the perception of some technologies varied considerably indicating that their use in practice might be dependent on specific factors. Due to the quantitative nature of the study, the authors could not definitely identify reasons for the varying perception so that the results remained "controversial” [23]. This was especially true for drones, which received a surprisingly negative feedback. Their dissemination in practice was found to be limited, too. ERIS, on the other hand, seemed to be more widespread in practice. Comparatively, they were also seen more positive but nevertheless found to be too complex for smaller departments. We hence decided to concentrate on these two rather unexpectedly assessed technologies to evaluate and identify possible causes for the perceptions.

\subsection{Emergency Response Information Systems}

ERIS aim at improving the coordination of emergency responses by providing a platform to gather and share relevant information on site (Figure 1). There are several types of ERIS being proposed in literature and practice. They differ in functionality and complexity.

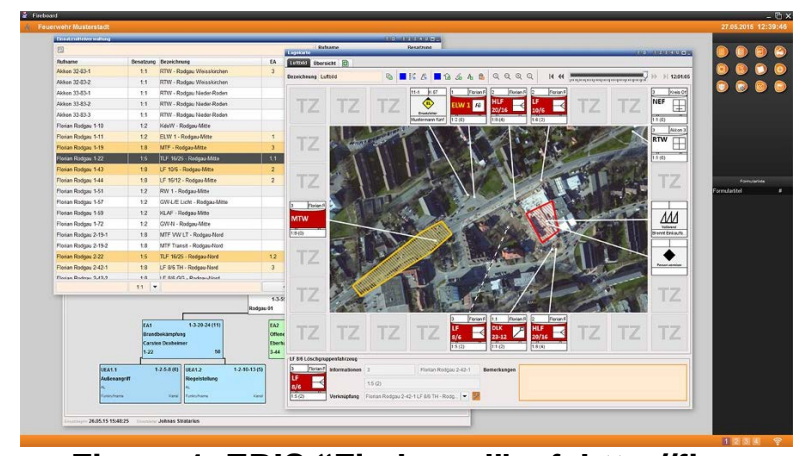

Figure 1. ERIS “Fireboard", cf. http://fireboard.net/en/fireboard/ 
The different types of ERIS particularly vary with respect to the way they obtain and utilize real-time data from the site. The functionality of basic ERIS is limited to the processing and presentation of information. The input of information usually is done manually [24]. This means that firefighters must feed the system with information during a response to benefit from its use. Many ERIS provided in practice can be assigned to this category [25, 26]. Apart from such comparatively simple systems, there also exist ERIS that capture real-time information using sensor networks [3, 27-31]. The captured information typically comprises the position of deployed units, tank levels of engines, outside temperature, wind direction etc. A third category of ERIS furthermore introduces decision support functionality [32, 33]. Those systems do not only present information to users. They also calculate and suggest possible decisions or commands based on the available information.

\subsection{Unmanned Aerial Vehicles}

To facilitate the exploration of an emergency site, literature frequently emphasizes the potential of UAVs. Both in academia and practice, various types of drones have been proposed to support firefighters (Figure 2).

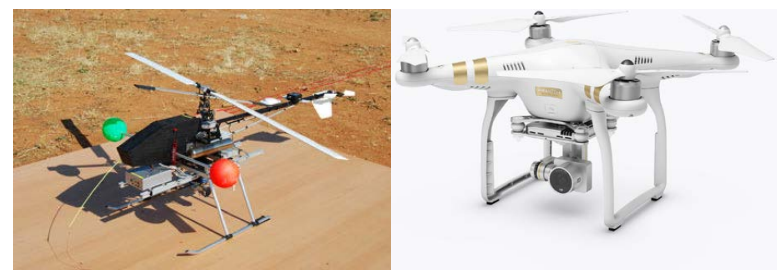

Figure 2. UAVs "TUB-H" [34] and "Phantom 3", cf. http://www.dji.com/phantom-3-pro

First, UAVs can be categorized by the tasks they are supposed to execute. A main application area for UAVs is the surveillance of the emergency site. Drones can, for instance, be used to detect and observe forest fires [35-39]. However, they can also be used for general reconnaissance tasks, which are required in any type of emergency operation [40, 41]. UAVs are also suggested to improve the on-site communication, for instance by establishing ad-hoc radio communication networks or increasing the range of existing ones [42, 43]. Drones can furthermore help during incidents with hazardous materials [44]. To measure the concentration of poisonous substances in the air, they are supposed to be more suitable than common on-ground measuring tools. Another task that is supposed to be supported is the search of victims or injured firefighters [34]. All in all, UAVs shall increase the commander's situation awareness.

UAVs can also be distinguished by their size and type of construction. In literature, rather small drones are supposed to be most suitable for FDs. They are typically designed as quad-, hexa-, or octocopters [44, 45]. Miniature helicopters are proposed as well [34, 37, 40]. In contrast, large drones - as used by the military - are proposed for specific tasks only. These types of UAVs resemble small planes [35]. A detailed categorization of drones based on their size can be found in literature [46].

UAVs also differ with respect to the way they are controlled by the user. On the one hand, there exist remotely controlled devices [36, 40]. They are piloted by an operator, who must either keep them in his/her sight or steer them by video transmission. On the other hand, autonomous UAVs have been suggested [37, 41, 44]. Such devices are for example assigned to a certain spot or area. A suitable route for reaching or covering this area is then computed and followed automatically.

Lastly, the number of drones deployed can be distinguished. Often, a single UAV is used [34, 36, 40], which can only provide information from a single point of view at any time. Other approaches require the deployment of multiple UAVs, so-called swarms [37, 39, 41, 44]. They can provide information from multiple locations within an area and are supposed to be especially suitable to surveil large areas or the spreading of poisonous gases.

\section{Research Method}

In new and emerging fields where little is known about the object of investigation, literature recommends employing qualitative research designs [47]. As shown in section 2, FITs mainly have been investigated from a technology rather than from a user perspective so far. Since little research exists that examines how FITs are perceived by their users, we decided to adopt an exploratory, qualitative research design. Doing so allowed us to gather in-depth insights into the perceived potential of the technologies. This research design also allowed us to gain an understanding of the reasons behind the perceptions. As emerging technologies are continuously adjusted and redeveloped, the reasons behind the perceptions of users are of practical interest as well and hence build an essential part of our research endeavor. Since emerging technologies furthermore often exist in different instantiations and not all participants might have the same understanding of a technology, we used the direct contact to the experts to make sure that they had a common understanding of the subject matter.

We decided to conduct semi-structured face-to-face interviews as they are considered the superior data collection technique for qualitative study designs [48]. Following a common, standardized interview guideline, semi-structured interviews shall ensure comparable results. But as the interviewer can adjust questions or ask for explanations if necessary, this interviewing form 
provides a greater breadth of results than rigorously structured interviews. Our interview guideline consisted of three parts. First, we asked for demographic information to gain insights into the interviewee's background and the FD. For instance, we asked for the number of firefighters working in the FD, the number of operations in one year, and the interviewee's qualifications. In the second part, we introduced the FITs to ensure common understanding. Afterward, we asked how the participants perceive the potential of the technologies. To identify positive and negative factors that influence the acceptance, we asked for perceived advantages, disadvantages and properties of the FITs affecting complexity. Also, we asked which requirements the FITs need to fulfill to be usable. During the third part, open questions were asked. For example, we wanted to know which technologies were already in use or intended to be introduced. Altogether, the interviews closely followed the guidelines given by Myers and Newman [49].

All interviewees were experts in the field. Generally, literature defines an expert as someone with privileged knowledge about the subject matter [50]. Regarding our research endeavor, an expert is someone who not only knows about the examined FITs but also has an extensive background in the way firefighters work and use such technologies on site. As experts typically are able to provide profound insights regarding the subject matter, their number can be rather low as long as they are selected carefully [50]. We decided to interview experts from the strategic, tactical, and operational command level of different FDs. The strategic command level consists of (assistant) fire chiefs responsible for principal matters and leading large-scale responses. The tactical command level is made of platoon leaders typically acting as incident commanders. The operational command level consists of squad leaders enforcing activities on site. In the role of command assistants, they will also be the ones to use the two examined technologies. With our strategy, we could hence gather perceptions from multiple perspectives and enhance the validity of the results. In total, we interviewed 21 firefighters that were nominated as experts according to the above-mentioned criteria by their FDs and had profound field experiences. The interviews were conducted in seven FDs distributed across Germany: two plant FDs, two professional FDs, and three voluntary FDs. They consisted of 70 to 900 firefighters and had 200 to 25.000 operations a year.

The gathered interview statements were analyzed for positive and negative perceptions as well as technology requirements. First, we used open coding techniques to identify recurrent statements that we grouped into topics. We then used in-vivo codes to name each topic with the denomination predominantly used by the experts. In so doing, we identified several factors that seem to determine the practical potential of ERIS and UAVs.

\section{Results and Discussion}

For each of the technologies, we describe the positive and negative factors as well as the existing requirements that have been emphasized by the interviewees. Aspects that have been mentioned by at least $33 \%$ of the participants are discussed in detail. To refer to individual interviewees, we numbered them consecutively.

\subsection{Emergency Response Information Systems}

Regarding the practical potential of ERIS, we identified seven positive, one neutral, 13 negative factors, and eight general requirements (cf. Table 1).

Table 1. Assessment of ERIS

\begin{tabular}{|c|c|c|c|}
\hline & Factor & $\mathbf{n}$ & $\%$ \\
\hline \multirow{7}{*}{ : } & Informational advantage & 14 & 67 \\
\hline & Increased capacity / documentation & 8 & 38 \\
\hline & Time advantage & 8 & 38 \\
\hline & Accuracy & 7 & 33 \\
\hline & Load removal from radio & 4 & 19 \\
\hline & Compactness & 2 & 10 \\
\hline & Structuring & 2 & 10 \\
\hline Neut. & Flexibility & 6 & 29 \\
\hline \multirow{13}{*}{ 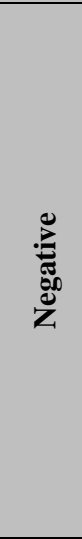 } & Decision-making complexity & 11 & 52 \\
\hline & Costs & 11 & 52 \\
\hline & Loss of competences & 10 & 48 \\
\hline & Resistance to change & 9 & 43 \\
\hline & Training effort & 8 & 38 \\
\hline & Lack of expressive power & 7 & 33 \\
\hline & Information overload & 7 & 33 \\
\hline & Personnel effort & 6 & 29 \\
\hline & Limited range of application & 3 & 14 \\
\hline & Maintenance / updating effort & 3 & 14 \\
\hline & Organizational effort & 3 & 14 \\
\hline & Less communication & 2 & 10 \\
\hline & Weight & 1 & 5 \\
\hline \multirow{8}{*}{ 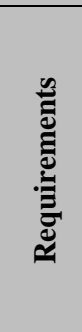 } & Intelligibility & 18 & 86 \\
\hline & Simplicity & 17 & 81 \\
\hline & Reliability & 12 & 57 \\
\hline & Robustness & 7 & 33 \\
\hline & Legal issues / privacy & 5 & 24 \\
\hline & Time restriction & 5 & 24 \\
\hline & Flexibility & 4 & 19 \\
\hline & Long lifespans in FDs & 2 & 10 \\
\hline
\end{tabular}

Positive. $67 \%$ of the experts found that ERIS offer an informational advantage: "A real benefit [...] will be reached once I have an electronic situation report that includes as much information as possible from systems that exist anyway. [...] Of course, you could extend this with sensor networks or decision support systems" (20). 
The automatic gathering of information using sensors is also seen positively: "Being able to see who is where at what point of time is an excellent basis to get an overview of an operation but also to assess the operation in case of accidents and to study what didn't work" (15).

An increased capacity and documentation was found positive by 38\%: "I can document the situation dynamically. With flipcharts, I always have the problem of changing or saving recordings" (3). "This would help the commander with respect to documentation, which is becoming ever more important due to legal issues" (7).

A time advantage was attested by 38\%: "Such a software is a wonderful supplement for a fast, transparent situation report" (2). Especially for sensor networks, the "real-time presentation" (20) was stated to be a benefit.

An increased accuracy was seen as advantageous by 33\% of the experts: "The huge advantage is that your situation reports are more accurate. So, I can coordinate or brief my units more accurately, as well" (14).

Negative. While the before-mentioned informational advantage could facilitate the decision-making, $52 \%$ of the experts found that a vast amount of information and a documentation of every decision could also make decision-making more complex: "Too many moving images in the decision-making room just hamper the decision-making" (20). "It documents everything. [...] Afterward, if the district attorney comes to investigate the cause of something that has gone wrong, this data can, of course, be inspected and used to interrogate or to hold responsible the decision-maker" (17). In addition, the potential influence of decision support systems was seen critically: "There is a danger that one might rely on things proposed by the system too quickly and that it is just an automated decision - but not necessarily the right one. [...]. I see that as a danger" (8).

$52 \%$ of the interviewees mentioned costs as a negative factor: "That will probably fail due to its cost" (10). This concern also applies to sensor networks: "Sensor technology would increase the costs of vehicles and equipment, which will not prevail, I think" (18).

As a specific problem of decision support systems, the potential loss of competences was addressed as a problem by 48\%: "For such things, I have my personnel. My team at the front is supposed to estimate and tell me, how things are going" (12). Introducing such systems would mean "a qualitative shift since I already have a manual assessment by the commander or the people in charge that would fall away" (19).

Especially for decision support systems, 43\% saw the resistance to change in their departments problematic: "This is a great thing, and you can see the tactical necessity, also the benefit. But if you have someone saying 'I don't want to use that', then he will not use it. So, you must convince your team to use the system” (2).
$38 \%$ of the experts also stated an increasing training effort: "The ones operating those systems must be trained and experienced in operating them" (10).

$33 \%$ also mentioned the lack of expressive power as a drawback of sensor networks: "Sensors are built for a certain physical unit. They can capture changes in those units, but nothing else. They can, for example, not capture if someone is in stress. So, there is the danger of getting values that are incomplete or do not necessarily match the reality" (9). This concern also applies to decision support systems: "There are so many parameters to be considered. I don't think that you could supply a decision support system with all that information. You will still need people with practical experience to estimate the situation” (2).

According to $33 \%$ of the experts, information overload is another disadvantage of sensor networks and decision support systems: "If there is a suggestion created for everything, I will have no time for anything but saying 'yes' or 'no' anymore” (10).

Requirements. $86 \%$ of the interviewees mentioned the intelligibility of the displayed information as a requirement: "[The display has to be organized] based on common knowledge. [...] That is a basic requirement. [...] The things displayed must look exactly as the things we had on the blackboard or on paper before" (19). "It needs to be organized in a way that you can process all necessary information at a single glance” (1).

$81 \%$ of the participants emphasized that ERIS must be simple and intuitively usable: "Concerning the handling, I demand that they are firefighter-proof” (13). "They have to make use of technology, which is known by nearly everybody" (4).

$57 \%$ emphasized reliability as a requirement: "Software solutions sometimes [...] don't work failure-free, which would be fatal during an operation" (3). "Equipping all firefighters with sensors makes me think of this: the more technology is built into a car, the more can break down" (11). "You cannot blindly rely on such systems. Actually, you always have to act on the assumption that such a system can crash" (21).

$33 \%$ of the interview partners also addressed robustness as an essential characteristic. Especially if devices are to be carried on site, they must withstand outdoor conditions: "You are not in an office, where everything is clean. If it is raining, it must still be working" (5).

\subsection{Unmanned Aerial Vehicles}

Regarding the practical potential of UAVs, we identified four positive factors, twelve negatives, and eight general requirements (cf. Table 2).

Positive. All experts found that UAVs can provide an informational advantage by expanding the commanders' perspective: "We are certainly lacking intelligence 
from above [...]. And that would definitely be beneficial" (2). "I could have a live picture from the distance. If I send in a firefighter, I can only hear what he reports [...] and don't have an overview of my own" (1).

$57 \%$ of the interviewees stated that drones provide a time advantage: "I'm probably faster with an UAV" (17). "Often there are no access points to an object so that you cannot see much from the ground. If you have an aerial view or a thermal image from above, you get a situational overview faster" (7).

$38 \%$ of the participants emphasized the currentness of data delivered by UAVs as another positive factor: "I can capture the current situation with an UAV. And not only the static situation, but the dynamic situation" (10). "Commonly, you will use Google maps excerpts which are one year, two years, perhaps only one day old. But they don't express the current situation" (13).

\section{Table 2. Assessment of UAVs}

\begin{tabular}{|c|c|c|c|}
\hline & Factor & n & $\%$ \\
\hline \multirow{4}{*}{ 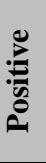 } & Informational advantage & 21 & 100 \\
\hline & Time advantage & 12 & 57 \\
\hline & Currentness of data & 8 & 38 \\
\hline & Safety & 4 & 19 \\
\hline \multirow{12}{*}{ 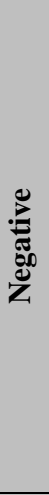 } & Personnel effort & 14 & 67 \\
\hline & Costs & 13 & 62 \\
\hline & Limited range of application & 12 & 57 \\
\hline & Training effort & 12 & 57 \\
\hline & Operation complexity & 11 & 52 \\
\hline & Maintenance / updating effort & 10 & 48 \\
\hline & Organizational effort & 10 & 48 \\
\hline & Evaluation effort & 4 & 19 \\
\hline & Resistance to change & 3 & 14 \\
\hline & Space requirements & 2 & 10 \\
\hline & Information overload & 2 & 10 \\
\hline & Decision-making complexity & 1 & 5 \\
\hline \multirow{8}{*}{ 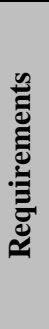 } & Robustness & 17 & 81 \\
\hline & Simplicity & 17 & 81 \\
\hline & Legal issues / privacy & 10 & 48 \\
\hline & Operating time & 8 & 38 \\
\hline & Reliability & 6 & 29 \\
\hline & Range & 3 & 14 \\
\hline & Loading capacity & 2 & 10 \\
\hline & Time restriction & 1 & 5 \\
\hline
\end{tabular}

Negative. $67 \%$ of the experts criticized the personnel effort to use UAVs: "If UAVs shall be available anytime, you need several people on every shift who can operate or fly these things. I see it in our department: personnel is scarce. [...] The question is who operates them" (17).

High costs were stated as another negative factor by 62\%: "Acquisition costs and operating costs. Operating a drone in an FD means providing multiple batteries for switching, which is an expensive part of such a device" (14). "If I wanted to make a safe aircraft out of it, the thing would become so expensive that you couldn't use it for such purposes anymore" (9).

$57 \%$ of the interviewees mentioned the limited range of application as a negative aspect: "I would [...] deploy it selectively and would not let it take off during tasks such as fighting room fires [...]. I don't think that I would rely on an UAV in those situations" (10). "How frequently will such a thing be deployed?" (5).

The high training effort required to operate UAVs is criticized by $57 \%$ of the experts: "If you need people who operate them - well then there will certainly be an according training effort" (19). "I find that problematic: not everyone can do that and you will definitely need people who have trained it" (14).

$52 \%$ of the interview partners also mentioned the operational complexity as a problem: "Airspace security must be considered. Especially in large-scale responses, where police and rescue helicopters are on the scene as well" (7). "Having smoke emission, I can easily get into some blind spots. [...] So, I need to know where to move, what the wind direction is, and so on" (13).

$48 \%$ of the interviewees named the maintenance or updating effort as a drawback: "If they are equipped with several sensor technologies [...] it will not only be an expensive, but also a high-maintenance device" (16).

The organizational effort was criticized by $48 \%$ : "You would need to establish a distinct group of people responsible for it" (1). Especially the deployment of autonomous UAVs is seen critical: "They will need an allocated air corridor; they will need a license" (8).

Requirements. Robustness was mentioned as a requirement by $81 \%$ of the experts, since UAVs would have to withstand weather and other extreme conditions in the incident area: "It would have to be able to fly in the rain [...] and it should be autonomous enough to compensate wind drifts" (1). "How close can I fly above a fire source without getting problems with the thermal lift? These things don't have much weight, so [...] they will quickly get problems with thermal lift" (12).

$81 \%$ stated simplicity as an essential factor since UAVs have "to be operated easily" (6). In particular, a certain degree of autonomy was desired: "I want to put it on the ground, specify the point of the disaster [...] and the flying altitude [...] and it should automatically approach the destination and deliver the image" (7).

$48 \%$ of the experts emphasized that legal issues and privacy concerns must be solved before introducing UAVs: "Legally unclear things like how I may use drones or what's happening with pictures I randomly record which may restrict people in their privacy" (8).

$38 \%$ demanded a long operating time: "Half an hour at least. If I must patrol a sector once or multiple times, it must stay in the air for quite some time” (5). 


\subsection{Discussion}

The interviewees found both FITs to deliver potential improvements. However, they also pointed to several potential drawbacks and constraints, which have to be fulfilled. All in all, each interview partner stated four positive factors, eight negative factors, and six requirements. We hence observed a rather diverse attitude, which contradicts unilaterally positive expectations that are often found in literature. The results rather emphasize that FITs are indeed delicate artifacts that have to be designed carefully and with acceptance-related factors closely kept in mind from the beginning.

In particular, the identified requirements seem to be critical success factors that ought to be fulfilled during the design of ERIS or UAVs for firefighters. We suppose that the acceptance of a specific technology can be significantly facilitated if the design fulfills the identified requirements. If a design fails to meet the stated requirements, its acceptance might be in jeopardy.

In comparison, it appears that the practical potential of ERIS is perceived as somewhat more positive than that of UAVs. Summing up the frequencies of positive and negative factors, the data contains 45 mentions of positive factors and 81 mentions of negative factors for ERIS. For UAVs, the data contains 45 mentions of positive factors and 94 mentions of negative factors. The ratio of positive and negative statements hence is slightly more positive for ERIS. As we cannot quantitatively express the relative influence of each factor on the acceptance, it only provides a first indication, though.

Table 3 shows factors that were mentioned both for ERIS and UAVs and compares the frequencies of mentions. We can conclude that UAVs were primarily seen as a means to gather information faster. However, they appear to be also perceived as rather expensive, requiring a high amount of personnel, and being limited in their range of application. Besides, simplicity, robustness and privacy were found to be important requirements that need to be fulfilled by UAVs. ERIS were found to also deliver an informational advantage. The added complexity during the decision-making process and the risk of introducing an information overload were found to be negative, though. We also found that the resistance to change might be higher for ERIS than for drones, which primarily support the gathering of data while ERIS have a direct influence on critical decisions. Accordingly, reliability and timing constraints were uttered more prominently for ERIS than for UAVs.

The results of our study corroborate and explain findings of a quantitative study that was recently conducted to examine the potential and the diffusion of emerging FITs [23]. That study showed that ERIS were both more widespread in use and perceived to have a greater potential to expedite the emergency response process than UAVs. The results of our study furthermore uncover the reasons behind these perceptions.

We also found indications that the attitude towards FITs might be influenced by resistance to change, which was mentioned for both technologies (Table 3). Obviously, firefighters are consciously reluctant to change established practices that have proven to be reliable. To some extent, this might explain why the FITs generally were viewed rather skeptically and the frequency of negative factors was higher than that of positive factors.

Altogether, the results indicate that introducing innovative FITs is a potentially complex topic. Even aspects which are perceived as beneficial at first might ultimately result in a drawback. For example, the most frequently stated positive factor of both ERIS and UAVs was an informational advantage. At the same time, however, it was feared that this advantage could lead to an information overload or raise the decision-making complexity. This shows that the design of innovative FITs requires a high amount of user involvement. A thorough evaluation by the users appears to be important to ensure that the technology will indeed support the firefighters during their work in the aspired way.

\section{Table 3. Comparison of ERIS and UAVs}

\begin{tabular}{|c|c|c|c|}
\hline & Factor & $\begin{array}{c}\% \\
\text { ERIS }\end{array}$ & $\begin{array}{c}\% \\
\text { UAVs }\end{array}$ \\
\hline \multirow{2}{*}{$\stackrel{8}{0}$} & Informational advantage & 67 & 100 \\
\hline & Time advantage & 38 & 57 \\
\hline \multirow{9}{*}{ 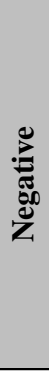 } & Costs & 52 & 62 \\
\hline & Training effort & 38 & 57 \\
\hline & Personnel effort & 29 & 67 \\
\hline & Limited range of application & 14 & 57 \\
\hline & Maintenance / updating effort & 14 & 48 \\
\hline & Organizational effort & 14 & 48 \\
\hline & Resistance to change & 43 & 14 \\
\hline & Decision-making complexity & 52 & 5 \\
\hline & Information overload & 33 & 10 \\
\hline \multirow{5}{*}{ 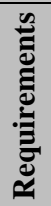 } & Simplicity & 81 & 81 \\
\hline & Robustness & 33 & 81 \\
\hline & Reliability & 57 & 29 \\
\hline & Legal issues / privacy & 24 & 48 \\
\hline & Time restriction & 24 & 5 \\
\hline
\end{tabular}

\subsection{Implications}

The provided insights into the potential of ERIS and UAVs have implications for academia and practice. Regarding academia, we provide a multifaceted overview of the benefits and risks that affect the potential acceptance of ERIS and UAVs. Next to that, we also identified requirements that the surveyed technologies have to fulfill in order to be usable in a practical setting. From a theoretical perspective, our results provide an initial 
set of acceptance factors for FITs. These factors can be used to evaluate the acceptance of FITs in more detail in quantitative studies. They furthermore contribute to the building of acceptance theories in this field. To arrive at a more general theory, future research will have to extend the amount of investigated FITs and to consolidate the identified factors, though. Next, the possible influence of command levels and FD types on the assessment of FITs should be investigated. A quantitative study could furthermore provide insights into the relative influence of the factors in comparison to each other, for instance by using path analyses or related methods.

Regarding practice, our work particularly has implications for FDs and FIT developers. The identified factors provide a means to assist FDs in contemplating the right questions when deciding on the acquisition of a FIT. For developers of FITs, our results can be employed as an instrument to evaluate their products and better adapt them to the needs of the FDs. In this context, the identified requirements might be of particular interest because they describe how the FITs ought to be designed to be more compatible to the way firefighters work.

\subsection{Limitations}

We have taken several precautions to ensure the validity of our results. To obtain comparable, unbiased data, we decided to conduct semi-structured interviews. By interviewing experts from different command levels, FD types, and regions, we tried to obtain a representative data set. During the coding stage, the team furthermore discussed the emerging codes repeatedly. Since the results stem from an analysis of qualitative data, they only constitute well-grounded assumptions, however. Ideally, they should be verified quantitatively. So far, we furthermore interviewed experts from German FDs only. Since the organization and the processes of FDs may differ, the results should not straightforwardly be transferred to other countries. The generalizability of our results instead remains to be validated, for instance by interviewing experts from different countries. Finally, we discussed only two FITs. Although the results of our study provide indications for relevant acceptance factors, they are not general enough to formulate a universal theory on the acceptance of FITs. To achieve such a goal, other types of FITs have to be examined as well.

At this stage, we also cannot yet say much about the relative influence that the identified factors have on the acceptance. The relative influence of the factors has to be analyzed more closely in quantitative studies. It may also vary depending on the scenario, in which a FIT is used. When responding to a major disaster, for instance, other factors might be important than during daily operations. Generally, researchers should also examine the practices of firefighters more intensively and formulate requirements and needs for FITs based on the identified use cases. Such endeavors could lead to further insights into desirable properties of FITs and complement the results of our study, which focused on evaluating FITs and hence is somewhat technology-centric in nature, too.

\section{Conclusion}

Although it is repeatedly proposed in literature to equip firefighters with innovative technologies, the practical potential of emerging FITs hardly has been in the focus of research. To contribute to the closure of this research gap, we presented the results of a qualitative study, in which we interviewed 21 German firefighters about the practical potential of ERIS und UAVs.

From the gathered data, we obtained rich insights into the aspects that facilitate or hinder the adoption of these FITs as well as the existing requirements. The results of our study hence provide a unique overview of factors that determine the acceptance of ERIS and UAVs. In contrast to the expectations, the practitioners' attitude towards these technologies appeared to be rather cautious. In particular, we encountered several concerns and constraints that can outweigh the expected benefits in practice if they are not managed carefully during the design and introduction of novel FITs.

While the presented results specifically apply to ERIS and UAVs, they call for an in-depth analysis and a more systematic consideration of acceptance-related factors when designing new FITs in general. It appears that emerging technologies are often arbitrarily used as a means to create new functionalities for emergency responders because of their desirable features. Such technology-driven approaches run a risk of neglecting the observation that information technologies are delicate artifacts for emergency responders, for which tight constraints and requirements have to be met.

To provide further insights into this particular field of application, future studies ought to verify our results in other regions and contexts. They should also evaluate the practical potential of additional emerging FITs such as unmanned ground vehicles or intelligent protective clothing. Based on such additional findings, it might be conceivable to derive a theory that explains the acceptance of emerging FITs. We hope that the results of our study can be a step into this direction.

\section{References}

[1] S. Mehrotra, C. Butts, D. Kalashnikov, N. Venkatasubramanian, R. Rao, G. Chockalingam, et al., "Project Rescue: Challenges in Responding to the Unexpected," in Proceedings of SPIE - The International Society for Optical Engineering, 2004, pp. 179-192. 
[2] M. Danielsson, "The cognitive structure of decision making tasks in major versus minor emergency response," in Proceedings of the Global Ergonomics Conference, 1998.

[3] L. Yang, R. Prasanna, and M. King, "On-Site Information Systems Design for Emergency First Responders," Journal of Information Technology Theory and Application, vol. 10, pp. 5-27, 2009.

[4] J. Webster and R. T. Watson, "Analyzing the Past to Prepare for the Future: Writing a Literature Review," MIS Quarterly, vol. 26, 2002.

[5] W. R. King and J. He, "Understanding the Role and Methods of Meta-Analysis in IS Research," Communications of the Association for Information Systems, vol. 16, pp. 665686, 2005.

[6] D. M. Neal, "Reconsidering the phases of disasters," International Journal of Mass Emergencies and Disasters, vol. 15, pp. 239-264, 1997.

[7] S. Ainuddin and J. K. Routray, "Institutional framework, key stakeholders and community preparedness for earthquake induced disaster management in Balochistan," Disaster Prevention and Management, vol. 21, pp. 22-36, 2012.

[8] R. Shaw, "Indian Ocean tsunami and aftermath: Need for environment-disaster synergy in the reconstruction process," Disaster Prevention and Management, vol. 15, pp. 5-20, 2006.

[9] D. L. Mallick, A. Rahman, M. Alam, A. S. M. Juel, A. N. Ahmad, and S. S. Alam, "Case study 3: Bangladesh floods in Bangladesh: A shift from disaster management towards disaster preparedness," IDS bulletin, vol. 36, pp. 53-70, 2005.

[10] M. Janssen, J. Lee, N. Bharosa, and A. Cresswell, "Advances in multi-agency disaster management: Key elements in disaster research," Information Systems Frontiers, vol. 12, pp. 1-7, 2010.

[11] N. Bharosa, J. Lee, and M. Janssen, "Challenges and obstacles in sharing and coordinating information during multi-agency disaster response: Propositions from field exercises," Information Systems Frontiers, vol. 12, pp. 49-65, 2010.

[12] N.-S. Lee, S. Hirschmeier, S. Müller, and L. J. Luz, "Enablers in Crisis Information Management: A Literature Review," presented at the 50th Hawaii International Conference on System Sciences, 2017.

[13] R. Power and J. Kibell, "The Social Media Intelligence Analyst for Emergency Management," presented at the 50th Hawaii International Conference on System Sciences, 2017.

[14] R. Rao, L. Plotnick, and S. R. Hiltz, "Supporting the Use of Social Media by Emergency Managers: Software Tools to Overcome Information Overload," presented at the 50th Hawaii International Conference on System Sciences, 2017.
[15] R. Subba and T. Bui, "Online Convergence Behavior, Social Media Communications and Crisis Response: An Empirical Study of the 2015 Nepal Earthquake Police Twitter Project," presented at the 50th Hawaii International Conference on System Sciences, 2017.

[16] K. Takahagi, T. Ishida, S. Noda, A. Sakuraba, N. Uchida, and Y. Shibata, "Proposal of the Fire Fighting Support System for the Volunteer Fire Company," presented at the 29th International Conference on Advanced Information Networking and Applications Workshops, 2015.

[17] J. H. Hong, J. Taylor, and E. T. Matson, "Natural Multilanguage Interaction between Firefighters and Fire Fighting Robots," presented at the International Joint Conferences on Web Intelligence and Intelligent Agent Technologies, 2014.

[18] M. Kozlovszky and D. Z. Pavlinic, "Environment and situation monitoring for firefighter teams," presented at the International Symposium on Computational Intelligence and Informatics, 2014.

[19] M. Klann and M. Geissler, "Experience Prototyping: A New Approach to Designing Firefighter Navigation Support," IEEE Pervasive Computing, vol. 11, pp. 68-77, 2012.

[20] L. Ramirez, T. Dyrks, J. Gerwinski, M. Betz, M. Scholz, and V. Wulf, "Landmarke: an ad hoc deployable ubicomp infrastructure to support indoor navigation of firefighters," Personal and Ubiquitous Computing, vol. 16, pp. 1025-1038, 2012.

[21] R. Ma and D. B. Kaber, "Situation awareness and workload in driving while using adaptive cruise control and a cell phone," International Journal of Industrial Ergonomics, vol. 35, pp. 939-953, 2005.

[22] M. R. Endsley, "Theoretical Underpinnings of Situation Awareness: A Critical Review," in Situation Awareness Analysis and Measurement, M. R. Endsley and D. J. Garland, Eds., ed Mahwah, NJ: Lawrence Erlbaum Associates, 2000.

[23] S. Schlauderer, S. Overhage, and J. Weidinger, "New Vistas for Firefighter Information Systems? Towards a Systematic Evaluation of Emerging Technologies from a Task-Technology Fit Perspective," presented at the 49th Hawaii International Conference on System Sciences, 2016.

[24] A. Monares, S. F. Ochoa, J. A. Pino, V. Herskovic, and A. Neyem, "MobileMap: A collaborative application to support emergency situations in urban areas," presented at the 13th International Conference on Computer Supported Cooperative Work in Design, 2009.

[25] N.N. (2017-06-06). Tablet Command - Tour. Available: http://tabletcommand.com/tour/

[26] N.N. (2017-06-06). Fireboard - Base System and addons. Available: http://fireboard.net/en/discoverfireboard/\#c1181 
[27] R. Granlund, H. Granlund, B. Johansson, and N. Dahlbäck, "The Effect of a Geographical Information System on Communication in Professional Emergency Response Organizations," presented at the 7th International Conference on Information Systems for Crisis Response and Management, 2010.

[28] A. Lewandowski, A. Schulz, R. Koch, and C. Wietfeld, "Mobile IT Applikation, vernetzte Sensoren und Kommunikationskonzepte zum Schutz der Einsatzkräfte bei der Feuerwehr," presented at the Workshop zur IT Unterstützung von Rettungskräften, 2009.

[29] K. Luyten, F. Winters, K. Coninx, D. Naudts, and I. Moerman, "A Situation-Aware Mobile System to Support Fire Brigades in Emergency Situations," in On the Move to Meaningful Internet Systems 2006, R. Meersman, Ed., ed: Springer Berlin, 2006, pp. 1966-1975.

[30] A. Panangadan, S. Monacos, S. Burleigh, J. Joswig, M. James, E. Chow, et al., "A system to provide real-time collaborative situational awareness by web enabling a distributed sensor network," presented at the the First ACM SIGSPATIAL Workshop, 2012.

[31] R. Prasanna, L. Yang, and M. King, "Evaluation of a software prototype for supporting fire emergency response," presented at the 8th International Conference on Information Systems for Crisis Response and Management, 2011.

[32] S. Shan and Q. Yan, Emergency Response Decision Support System. Singapore: Springer Singapore, 2017.

[33] K. Kalabokidis, G. Xanthopoulos, P. Moore, D. Caballero, G. Kallos, J. Llorens, et al., "Decision support system for forest fire protection in the Euro-Mediterranean region," European Journal of Forest Research, vol. 131, pp. 597-608, 2012.

[34] I. Maza, F. Caballero, J. Capitán, J. R. Martínez-de-Dios, and A. Ollero, "Experimental Results in Multi-UAV Coordination for Disaster Management and Civil Security Applications," Journal of Intelligent \& Robotic Systems, vol. 61, pp. 563-585, 2011.

[35] I. Reusen, N. Lewyckyj, S. Adriaensen, J. Biesemans, J. Everaerts, and S. Kempenaers, "Near-real-time forest fires monitoring system: case study with a manned aerial vehicle within the OSIRIS project," in FOREST FIRES 2008, J. de las Heras, C. A. Brebbia, D. Viegas, and V. Leone, Eds., ed: WIT, 2008, pp. 145-152.

[36] C. Barrado, R. Messeguer, J. Lopez, E. Pastor, E. Santamaria, and P. Royo, "Wildfire monitoring using a mixed air-ground mobile network," IEEE Pervasive Computing, vol. 9, pp. 24-32, 2010.

[37] L. Merino, F. Caballero, J. R. Martínez-de-Dios, I. Maza, and A. Ollero, "An Unmanned Aircraft System for Automatic Forest Fire Monitoring and Measurement," Journal of Intelligent \& Robotic Systems, vol. 65, pp. 533-548, 2012.
[38] H. Cruz, M. Eckert, J. Meneses, and J.-F. Martinez, "Efficient Forest Fire Detection Index for Application in Unmanned Aerial Systems (UASs)," Sensors, vol. 16, 2016.

[39] K. A. Ghamry and Y. Zhang, "Cooperative control of multiple UAVs for forest fire monitoring and detection," presented at the International Conference on Mechatronic and Embedded Systems and Applications, 2016.

[40] M. van Persie, A. Oostdijk, J. Fix, M. C. van Sijl, and L. Edgardh, "Real-time UAV based geospatial video integrated into the fire brigades crisis management GIS system," International archives of the photogrammetry, remote sensing and spatial information sciences, vol. 38, pp. 173-175, 2011.

[41] M. Quaritsch, R. Kuschnig, H. Hellwagner, B. Rinner, A. Adria, and U. Klagenfurt, "Fast aerial image acquisition and mosaicking for emergency response operations by collaborative UAVs," presented at the 8th International Conference on Information Systems for Crisis Response and Management, 2011.

[42] C. Christmann and E. Johnson, "Design and Implementation of a Self-configuring Ad-hoc Network for Unmanned Aerial Systems," presented at the AIAA Infotech@Aerospace 2007 Conference and Exhibit, 2007.

[43] D. Hague, H. Kung, and B. Suter, "Field Experimentation of Cots-Based UAV Networking," presented at the IEEE Military Communications Conference, 2006.

[44] N.N., "Project AirShield: Description of the Project AirShield and its results in the final demonstration at Falck RISC Rotterdam," microdrones GmbH, 2012.

[45] C. Yuan, Y. Zhang, and Z. Liu, "A survey on technologies for automatic forest fire monitoring, detection, and fighting using unmanned aerial vehicles and remote sensing techniques," Canadian Journal of Forest Research, vol. 45, pp. 783-792, 2015.

[46] A. C. Watts, V. G. Ambrosia, and E. A. Hinkley, "Unmanned Aircraft Systems in Remote Sensing and Scientific Research: Classification and Considerations of Use," Remote Sensing, vol. 4, pp. 1671-1692, 2012.

[47] A. Rubin and E. R. Babbie, Essential Research Methods for Social Work. Belmont, CA: Brooks Cole, 2006.

[48] R. K. Yin, Case Study Research: Design and Methods, 5 ed.: SAGE, 2014.

[49] M. D. Myers. (2015-05-23). Qualitative Research in Information Systems. Available: http://www.qual.auckland.ac.nz/

[50] A. Bogner, B. Littig, and W. Menz, Interviewing Experts: Palgrave Macmillan, 2009. 